\title{
Placental $L P L$ Gene Expression Is Increased in Severe Intrauterine Growth-Restricted Pregnancies
}

\author{
SILVIA TABANO, GIOIA ALVINO, PATRIZIO ANTONAZZO, FRANCESCA R. GRATI, MONICA MIOZZO, AND IRENE CETIN
}

Department of Medicine Surgery and Dentistry [S.T., F.R.G., M.M.], University of Milano, 20125 Milano, Italy; Institute of Obstetrics and Gynecology II [G.A., PA, I.C.], University of Milano, 20122 Milano, Italy; IRCCS Fondazione Policlinico, Mangiagalli,

Regina Elena [I.C.], 20122 Milano, Italy

\begin{abstract}
Intrauterine growth restriction (IUGR) is associated with reduced placental supply of nutrients to the fetus. Lipoprotein lipase (LPL) mediates the hydrolysis of triglycerides from maternal lipoproteins to obtain fatty acids. Here, we tested the hypothesis that placental $L P L$ gene expression level is altered in pregnancies complicated by IUGR. To this purpose, 28 IUGR fetuses were identified during pregnancy and divided in two groups: 7 M-IUGR ["mild" IUGR, with normal umbilical artery pulsatility index (PI)] and 21 S-IUGR ("severe" IUGR, with abnormal PI). Moreover, 10 out of 28 IUGR pregnancies were associated with preeclampsia. Controls were 19 normal pregnancies delivering appropriate for gestational age (AGA) fetuses. Relative real-time quantification of $L P L$ was carried out in RNA from placental chorionic villi by the $\Delta \Delta \mathrm{Ct}$ method, using $\beta$-actin as normalizing gene. Placental $L P L$ mRNA expression levels were significantly higher in IUGR than in AGA. In particular, significantly higher values were observed in S-IUGR, independent from the concomitant association with preeclampsia. No significant relationship was observed between placental $L P L$ mRNA expression levels or gestational age. In conclusion, placental $L P L$ mRNA gene expression is increased in severe IUGR, characterized by enhanced vascular placental resistances and alterations of placental nutrient transport. (Pediatr Res 59: 250-253, 2006)
\end{abstract}

$I^{\prime}$ UGR is a condition characterized by reduced supply of nutrients to the fetus from the uteroplacental unit (1), resulting from both placental insufficiency and inadequate maternal adaptation to pregnancy. The mechanism of transport of fatty acids across the placenta is not completely understood, with the placenta preferentially exchanging long-chain polyunsaturated fatty acids (LC-PUFA) $(2,3)$. Cellular uptake and intracellular translocation of nonesterified fatty acids have been proposed as part of a multistep process mediated by different membrane-associated and cytoplasmic proteins (4). Essential fatty acids are mainly provided to the placenta as triglycerides, carried by maternal triglyceride-rich lipoproteins $(5,6)$. They are taken up by lipoproteins receptors; subsequently, lipase activities mediate their hydrolysis to obtain fatty acids (5). LPL acts at one of the initial steps in transplacental transfer of FFA: it is involved in the hydrolysis of

Received July 20, 2005; accepted October 25, 2005.

Correspondence: Irene Cetin, M.D., Institute of Obstetrics and Gynecology, Luigi Mangiagalli, via della Commenda 12, 20122 Milano Italy; e-mail: Irene.Cetin@unimi.it Research supported by a European Union grant: PERILIP, contract number QLRT2001-00138. triglycerides present in chylomicrons and VLDL to generate fatty acids, a source of energy for the fetus (7). FFA can be subsequently esterified or oxidized, until they are transferred by facilitated diffusion to the fetus via fatty acid binding proteins (8). In this study, we measured $L P L$ mRNA abundance in the placenta, because it is known that this gene can be regulated at the transcriptional level (9). To our knowledge, $L P L$ mRNA expression levels have never been evaluated in the placenta. Nevertheless, recently, LPL protein activity was evaluated in MVM of syncytiotrophoblasts from normal and IUGR placentas (10), and the results were consistent with a lower activity of LPL in complicated pregnancies. In contrast, higher lipolytic activity has been reported in placental tissue homogenates of IUGR pregnancies (11). This discrepancy could be due to the different placental tissue analyzed, inasmuch as distinct lipase activities are expressed in different cellular types. Alterations of $L P L$ expression could be associated with changes in FFA placental exchange and might contribute to the abnormal lipid profile reported in fetal plasma of pregnancies characterized by IUGR (12). Here, we tested the hypothesis that IUGR pregnancies present a change in placental $L P L$ gene expression. In addition, we investigated whether the amount of this variation correlates with severity of placental insufficiency evaluated by umbilical artery Doppler velocimetry as an index of increased placental vascular resistance and by the presence of preeclampsia.

\section{SUBJECTS AND METHODS}

Subjects. Placentas were collected from IUGR and AGA pregnancies. All pregnancies were singleton and gestational age was calculated from last menstrual period and confirmed by ultrasound performed before 20 wk gestation. Fetuses with major malformations and/or chromosomal abnormalities were excluded from the study. IUGR was defined during pregnancy as 1) a percentile reduction of the abdominal circumference greater than $40 \%$ compared with the previous measurements by ultrasound (13) and 2) a birth weight below the 10th percentile (according to Italian standards for birth weight and gestational age) (14). We defined IUGR severity on the basis of Doppler velocimetry of the umbilical artery (15): 7 pregnancies with M-IUGR showed normal PI; 21 cases of S-IUGR showed abnormal PI. Ten out of 28 IUGR cases (2 M-IUGR and 8 S-IUGR) were associated with preeclampsia,

Abbreviations: AGA, appropriate for gestational age; IUGR, intrauterine growth restriction; LPL, lipoprotein lipase; M-IUGR, mild intrauterine growth restriction; MVM, microvillous plasma membranes; PI, pulsatility index; S-IUGR, severe intrauterine growth restriction

DOI: 10.1203/01.pdr.0000199441.62045.a1 
defined as blood pressure $>140 / 90 \mathrm{~mm} \mathrm{Hg}$ on two or more occasions, with a rise in diastolic blood pressure $>25 \mathrm{~mm} \mathrm{Hg}$ occurring after 20 wk of gestation, together with proteinuria $(>0.3 \mathrm{~g} / 24 \mathrm{~h}$ or $\geq 3+$ on dipstick testing) (16). Nineteen control pregnancies (AGA) were recruited among women who had healthy term neonates with birth weight between the 10th and 90th percentiles according to Italian standards (14). All women delivered by cesarean section in the absence of labor. Indications for cesarean section were breech or repeat cesarean section in the control group; pregnancy was terminated by cesarean section in the interest of the fetus in the IUGR group. Informed consent was obtained from all patients and the ethical committee of the Medical Faculty, University of Milan, granted approval. The characteristics of the population analyzed are summarized in Table 1 .

Immediately after delivery, a sample of placenta was taken from the fetal side. This was obtained by cutting of different portions (at least four) of the placenta and by pooling them together for the analysis. After washing with PBS solution (Dulbecco's phosphate buffered solution, Euroclone-PeroMilano, Italy) to eliminate residual blood, the sample was minced and microscopically inspected to select chorionic villi and remove the possible contaminating fragments of maternal decidua. Chorionic villi were stored in RNAlater medium within $30 \mathrm{~min}$ from sampling (Ambion, Austin, TX) and, following the manufacturer's recommendations, kept for $24 \mathrm{~h}$ at $+4^{\circ} \mathrm{C}$, and then at $-20^{\circ} \mathrm{C}$ until RNA extraction.

RNA extraction and cDNA synthesis. Total RNA was extracted by a single-step guanidinium-phenol-chloroform method. Potentially contaminating DNA was removed from the samples using a specific DNA-free kit (Ambion). The final concentration was spectrophotometrically determined and the integrity was verified by $0.4 \%$ agarose gel electrophoresis. Following the manufacturer's guidelines, $1 \mu \mathrm{g}$ of RNA was reverse transcribed in a final volume of $50 \mu \mathrm{L}$, containing $1 \times \mathrm{RT}$ buffer, $\mathrm{MgCl}_{2} 5.5 \mathrm{mM}, 500 \mu \mathrm{M}$ each dNTP, random hexamers $2.5 \mu \mathrm{M}, 0.4 \mathrm{U} / \mu \mathrm{L}$ of Rnase inhibitor, and 1.25 $\mathrm{U} / \mu \mathrm{L}$ MultiScribe Reverse Transcriptase (TaqMan Reverse Transcription Reagent, Applied Biosystems, Foster City, CA). The obtained cDNA was stored at $-20^{\circ} \mathrm{C}$ until use.

Real-time PCR. PCR reactions were performed using the ABI Prism 7700 Sequence Detection System (Applied Biosystems). Two microliters of cDNA were added to a PCR reaction mix, composed of $1 \times$ TaqMan Universal PCR Master Mix, $300 \mathrm{nM}$ TaqMan Probe (Applied Biosystems), $200 \mathrm{nM}$ each primer, and distilled water to a final volume of $25 \mu \mathrm{L}$. LPL expression levels were calculated relating to the housekeeping gene $\beta$-actin, chosen because of its stable expression throughout gestation (17). Each experiment was carried out in triplicate. The coefficient of variation of $\beta$-actin was $3.1 \%$ in AGA and $1.7 \%$ in IUGR placentas. The PCR thermal profile consisted of 2 min at $50^{\circ} \mathrm{C}$ and $10 \mathrm{~min}$ at $95^{\circ} \mathrm{C}$, followed by 40 cycles at $95^{\circ} \mathrm{C}$ for $15 \mathrm{~s}$ and $60^{\circ} \mathrm{C}$ for 1 $\min$. Data were analyzed using the Sequence Detection Systems 1.9 software (Applied Biosystems).

Primers and probes. Primers and probes were chosen using Primer Express 2.0 software (Applied Biosystems). Primers were as follows: LPL-F: 5'-TTG TGA AAT GCC ATG ACA AGT CT-3' and LPL-R: 5'-CAT GCC GTT CTT TGT TCT GTA GA-3', located in exons 9 and 10 of LPL gene, respectively. $\beta$-actin-F: $5^{\prime}$-GCG AGA AGA TGA CCC AGA TC-3' and $\beta$-actin R: $5^{\prime}$-CCA GTG GTA CGG CCA GAG G- ${ }^{\prime}$, mapped in exons 3 and 4 of $\beta$-actin, respectively. LPL TaqMan Probe (exons 9 and 10): 5'(FAM)TGA ATA AGA AGT CAG GCT GAA ACT GGG GC-(TAMRA) 3'; $\beta$-actin TaqMan Probe (exon 4): 5'(FAM)-CCA GCC ATG TAC GTT GCT ATC CAG GC-(TAMRA) 3'.

Statistical analysis. The relative $L P L$ gene expression levels were calculated using the previously described $2^{-\Delta \Delta \mathrm{Ct}}$ method (18). After testing for normal distribution, statistical analyses were conducted using the unpaired two-tailed $t$ tests, with $p<0.05$ as the significant level.

\section{RESULTS}

LPL expression study. Placental $L P L$ mRNA expression levels were significantly higher in IUGR (mean value: 12.88 arbitrary units) than in AGA (mean value: 3.26 arbitrary units) $(p<0.05)$. Figure 1 displays placental LPL mRNA expression levels in AGA and IUGR in relation to gestational age; as shown, there is no difference in $L P L$ expression related to gestational age. In our population, $L P L$ mRNA expression values did not correlate with maternal, placental, or fetal weight, nor with oxygen saturation or $\mathrm{Po}_{2}$ (data not shown). Dividing IUGR in different groups of increasing severity (Fig. 2), significantly higher levels of $L P L$ mRNA ( $p<0.05$ versus AGA) were observed in S-IUGR placentas, independently from the concomitant association with preeclampsia [mean value: 8.37 a.u. (arbitrary units) in S-IUGR without and 25.71 a.u. in S-IUGR with preeclampsia] compared with AGA ones (mean value: 3.26 a.u.). Significant increase in $L P L$ expression levels was not observed in M-IUGR (mean value: 3.16 a.u. in M-IUGR without and 1.66 a.u. in M-IUGR with preeclampsia).

\section{DISCUSSION}

The present study was aimed to investigate whether IUGR pregnancies are associated with altered expression levels of placental $L P L$ and to determine a possible correlation between different degrees of IUGR and the amount of $L P L$ mRNA. The regulation of $L P L$ gene expression is complex, occurring at transcriptional, translational, or posttranslational level, in a tissue-specific manner. In particular, in this study, mRNA levels were measured, inasmuch as it is known that LPL functions can be regulated modulating mRNA synthesis, thanks to the existence of cis-acting regulatory elements, located in the $5^{\prime}$ flanking region of the human $L P L$ gene (9). The fetus needs mostly essential fatty acids and their derivatives, LC-PUFA, for rapid incorporation into structural lipids of the brain, where they maintain the fluidity, permeability, and structure of membranes and play an important role in brain development and visual function (19). LPL is an enzyme involved in maternal lipoprotein hydrolysis, thus mediating

Table 1. Clinical features of the study subjects: IUGR are divided in two subgroups ( $M$ and S) according to umbilical arterial pulsatility index

\begin{tabular}{|c|c|c|c|}
\hline & AGA $(n=20)$ & M-IUGR $(n=6)$ & S-IUGR $(n=21)$ \\
\hline \multicolumn{4}{|l|}{ Mother } \\
\hline Maternal age (y) & $32.5(25-40)$ & $33.0(29-36)$ & $32.9(20-38)$ \\
\hline Pregravidic weight $(\mathrm{k})$ & $56.2(49-68)$ & $56.3(49-63)$ & $64.6 * *(49-80)$ \\
\hline Gestational age (wk of gestation) & $38+6(38-40+5)$ & $35+6^{*}(33-37+4)$ & $32+3^{*}(27+2-37+3)$ \\
\hline \multicolumn{4}{|l|}{ Conceptus } \\
\hline Fetal weight $(\mathrm{g})$ & $3162.2(1970-3920)$ & $1705.7 *(1250-2430)$ & $1317.7 *(451-2340)$ \\
\hline
\end{tabular}

Values are expressed as mean (range).

$* p$ Value $v s$ AGA $<0.01$

$* * p$ Value $v s$ AGA $<0.05$ 


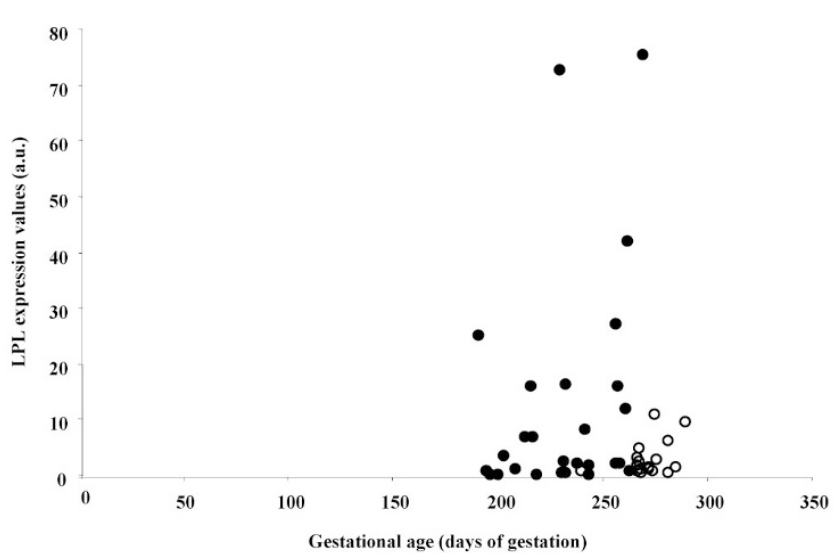

Figure 1. $L P L$ expression values in relation to gestational age (IUGR, black circles; AGA, open circles).

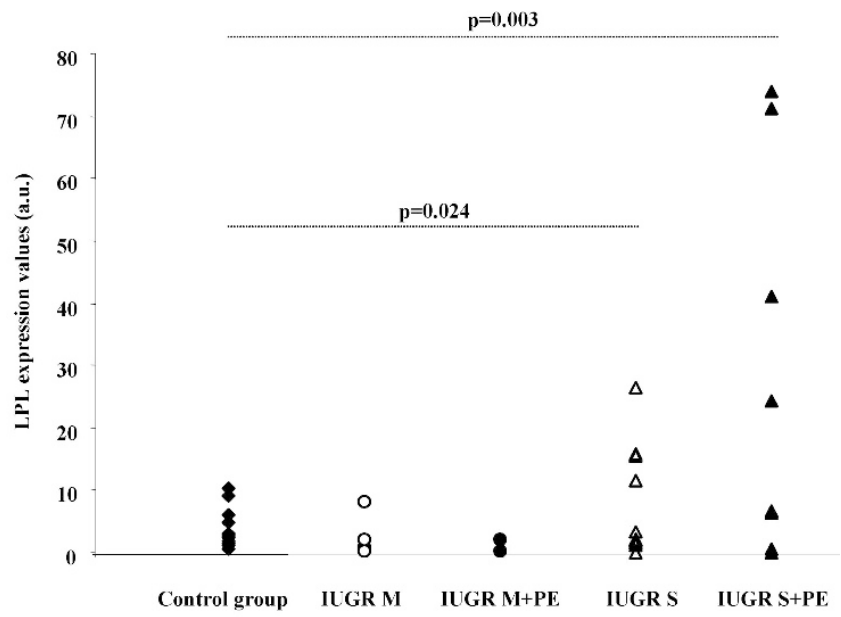

Figure 2. $L P L$ expression values of samples analyzed. IUGR are divided in four subgroups composed of 4 M-IUGR, 2 M-IUGR with preeclampsia (PE), 12 S-IUGR, and 9 S-IUGR with preeclampsia and compared with 20 AGA.

the first-limiting step for fatty acids availability from the mother to the developing fetus. Results presented here are in agreement with those previously reported by Biale et al. (11), which described increased LPL activity in placentas from IUGR and severe preeclamptic pregnancies compared with AGA ones. They also found an inverse relationship between LPL and intracellular lipase activities, with an increase in LPL and a decrease in intracellular lipase activities in IUGR as well as in preeclamptic pregnancies. In addition, in our study we describe an increased $L P L$ mRNA expression only in S-IUGR cases with abnormal umbilical blood flow, whereas no differences were observed between IUGR with normal umbilical PI and control placentas. A possible explanation for an increased $L P L$ expression level in S-IUGR placentas compared with AGA placentas could be that, in placental insufficiency, $L P L$ mRNA undergoes a lower degradation rate than in AGA, resulting in a higher mRNA stability and an increased gene expression. However, this is quite unlikely because S-IUGR are more likely to be associated with a condition of stress and hypoxia (15), which is characterized by apoptosis and, therefore, high mRNA degradation rate. Moreover, although $L P L$ expression was increased in S-IUGR independently from the association with preeclampsia, we observed the highest levels in S-IUGR with preeclampsia. Mothers of the S-IUGR group were also significantly heavier than the other two groups, although no relationship was observed between maternal body size and $L P L$ placental expression. The possible role of maternal body size and preeclampsia could be associated with changes in maternal lipoprotein concentrations and maternal systemic inflammatory effect influencing placental LPL expression (20).

One potential bias of our study is that IUGR pregnancies were delivered at an earlier gestational age than controls, because it is very rare to obtain placental samples from normal pregnancies delivered preterm for indications other than fetal. However, we did not observe any relationship with gestational age and we report high levels of $L P L$ mRNA also in IUGR at similar gestational ages than controls. In contrast to our results, Magnusson et al. (10) previously reported reduced LPL activity in placental MVM of preterm IUGR cases compared with age-matched preterm controls. To explain this discrepancy, we can speculate that, also in the presence of reduced LPL activity, LPL mRNA expression might be increased as a compensatory mechanism. Moreover, LPL is only responsible for $20 \%$ of the lipolytic activity of the MVM placental cellular type, whereas the major role is attributable to a second distinct lipase, as previously demonstrated (21). LPL activity becomes more important when, as in our study, placental homogenates with different cellular types are considered. At the moment, the site of production of LPL in the placenta is not known. Recent data report $L P L$ mRNA expression in the syncytiotrophoblast, whereas the product of the gene is present in both syncytiotrophoblast and endothelial cells (22).

In conclusion, this study indicates that $L P L$ mRNA expression is increased in severe forms of IUGR, characterized by placental insufficiency with abnormal umbilical PI values and preeclampsia. This could represent a compensatory mechanism of the placenta when the transfer of fatty acids is altered in chronically deprived fetuses.

\section{REFERENCES}

1. Pardi G, Marconi AM, Cetin I 2002 Placental-fetal interrelationship in IUGR fetuses-a review. Placenta 23:S136-S141

2. Haggarty P, 2002 Placental regulation of fatty acid delivery and its effect on fetal growth-a review. Placenta 23:S28-S38

3. Hendrickse W, Stammers JP, Hull D 1985 The transfer of free fatty acids across the human placenta. Br J Obstet Gynaecol 92:945-952

4. Campbell FM, Bush PG, Veerkamp JH, Dutta-Roy AK 1998 Detection and cellular localization of plasma membrane-associated and cytoplasmic fatty acid-binding proteins in human placenta. Placenta 19:409-415

5. Herrera E, 2002 Implications of dietary fatty acids during pregnancy on placental, fetal and postnatal development—a review. Placenta 23:S9-S19

6. Preiss-Landl K, Zimmermann R, Hammerle G, Zechner R 2002 Lipoprotein lipase: the regulation of tissue specific expression and its role in lipid and energy metabolism. Curr Opin Lipidol 13:471-481

7. Goldberg IJ, Merkel M 2001 Lipoprotein lipase: physiology, biochemistry, and molecular biology. Front Biosci 6:D388-D405

8. Dutta-Roy AK 2000 Transport mechanisms for long-chain polyunsaturated fatty acids in the human placenta. Am J Clin Nutr 71:315S-322S

9. Previato L Parrott CL,Santamarina-Fojo S, Brewer HB 1991 Transcriptional regulation of the human lipoprotein lipase gene in 3T3-L1 adipocytes. J Biol Chem 266:18958-18963

10. Magnusson AL, Waterman IJ, Wennergren M, Jansson T, Powell TL 2004 Triglyceride hydrolase activities and expression of fatty acid binding proteins in the human placenta in pregnancies complicated by intrauterine growth restriction and diabetes. J Clin Endocrinol Metab 89:4607-4614 
11. Biale Y 1985 Lipolytic activity in the placentas of chronically deprived fetuses. Acta Obstet Gynecol Scand 64:111-114

12. Cetin I, Giovannini N, Alvino G, Agostoni C, Riva E, Giovannini M, Pardi G 2002 Intrauterine growth restriction is associated with changes in polyunsaturated fatty acid fetal-maternal relationships. Pediatr Res 52:750-755

13. Todros T, Ferrazzi E, Groli C, Nicolini U, Parodi L, Pavoni M, Zorzoli A, Zucca S 1987 Fitting growth curves to head and abdomen measurements of the fetus: a multicentric study. J Clin Ultrasound 15:95-105

14. Parazzini F, Cortinovis I, Bortolus R, Fedele L 1991 Standards of birth weight in Italy [in Italian]. Ann Ostet Ginecol Med Perinat 112:203-246

15. Pardi G, Cetin I, Marconi AM, Lanfranchi A, Bozzetti P, Ferrazzi E, Buscaglia M, Battaglia FC 1993 Diagnostic value of blood sampling in fetuses with growth retardation. N Engl J Med 328:692-696

16. Redman CW, Jefferies M 1988 Revised definition of pre-eclampsia. Lancet 1:809-812

17. Lacey HA, Nolan T, Greenwood SL, Glazier JD, Sibley CP 2005 Gestational profile of $\mathrm{Na}+/ \mathrm{H}+$ exchanger and $\mathrm{Cl}-/ \mathrm{HCO}^{-}$anion exchanger mRNA expression in placenta using real-time QPCR. Placenta 26:93-98

18. Livak K 1997 Comparative Ct method. ABI Prism 7700 Sequence Detection System PE Applied Biosystems User Bulletin 2:11-15

19. Haggarty P 2004 Effect of placental function on fatty acid requirements during pregnancy. Eur J Clin Nutr 58:1559-1570

20. Ramsay JE, Ferrell WR, Crawford L, Wallace AM, Greer IA, Sattar N 2004 Divergent metabolic and vascular phenotypes in pre-eclampsia and intrauterine growth restriction: relevance of adiposity. J Hypertens 22:2177-2183

21. Waterman IJ, Emmison N, Sattar N, Dutta-Roy AK 2000 Further characterization of a novel triacylglycerol hydrolase activity ( $\mathrm{pH} 6.0$ optimum) from microvillous membranes from human term placenta. Placenta 21:813-823

22. Lindegaard ML, Olivecrona G, Christoffersen C, Kratky D, Hannibal J, Petersen BL, Zechner R, Damm P, Nielsen LB 2005 Endothelial and lipoprotein lipases in human and mouse placenta. J Lipid Res 46:2339-2346 